\title{
Experimental Characterization of Drobot: Towards Closed-Loop Control
}

\author{
Nathalie Majcherczyk ${ }^{1}$, Kanty Rabenorosoa ${ }^{2}$, Cédric Clévy ${ }^{2}$, Rosica Mincheva ${ }^{3}$, \\ Jean-Marie Raquez ${ }^{3}$, Marc Viallon ${ }^{4}$, Massimo Mastrangeli ${ }^{1}$ and Pierre Lambert ${ }^{1}$
}

\begin{abstract}
This paper presents advances in the development of a microrobotic platform actuated by three liquid droplets in a gaseous environment (Drop-bot or Drobot). Drobot builds on the bubble robotics concept earlier introduced by Lenders [1]. A new platform design is described allowing three actuated degrees of freedom : one translational (vertical) and two rotational (tilt). The platform-supporting droplets are generated by pushing liquid out of a tank through linear actuators. Preliminary kinematic (output/input ratio), static (compliance) and dynamic (response time and evaporation time) characterizations of Drobot are reported, as well as promising experiments with an ionic liquid, whose negligible volatility is suitable for vacuum or hot environments. Additionally, a method to deduce the platform attitude from the measurement of the electrical resistance of the droplets is discussed. This work contributes toward the automatic control of the platform, which will be fully addressed in a following publication.
\end{abstract}

\section{INTRODUCTION}

Microassembly deals with the assembly of submillimetric components [2]. Typical microassembly operations-such as gripping, moving, placing, and releasing microcomponents to defined locations - are affected by forces inherent to the microscale. Due to scaling laws, such forces can produce effects significantly different than at macroscale [2], [3]. By exploiting these effects, innovative devices can be designed to address relevant problems in microassembly automation. Eminent among such problems is the need to compensate for the absence at microscale of inherent elasticity in brittle materials such as e.g. silicon. Structural compliance could accomodate positioning and manufacturing errors by avoiding high contact forces and severe damage of handled components. The need for compliance was already pinpointed in literature. A millimetric assembly table suspended by three helical springs was developed in [4]. A modular, compliant platform for microassembly with integrated force measurement capabilities was proposed in [5]. The concept of a compliant table enabled by capillary effects was then introduced by Lenders [1], and further developed to handle components submerged in liquid using bubbles as actuators (Bubble-bot or Ro-bulle in French) [6], [7]. Compliant microrobotics falls into two emerging domains : bubble

\footnotetext{
${ }^{1}$ N. Majcherczyk, M. Mastrangeli and P. Lambert are with the BEAMS Department, École Polytechnique de Bruxelles, Université Libre de Bruxelles, 1050 Brussels, Belgium pierre. lambert@ulb. ac.be

${ }^{2} \mathrm{~K}$. Rabenorosoa and C. Clévy are with the AS2M Dept., FEMTO-ST, 25000 Besancon, France kanty.rabenorosoa@femto-st. fr

${ }^{3}$ J.-M. Raquez and R. Mincheva are with the Laboratory of Polymeric and Composite Materials, Université de Mons, B-7000 Mons, Belgium

${ }^{4} \mathrm{M}$. Viallon is with PFT Proto-micro, Lycée Edgar Faure, 25500 Morteau, France
}

and droplet robotics (in liquid and gaseous environments, respectively), and soft robotics.

The former-related to fluidic assembly [8] as well as to surface-tension-based microrobotics [3] -has seen for instance the use of oscillating bubbles to propel swimming robots [9], [10], of air bubbles to create Marangoni stresses driving cells in bulk liquid [11] and of opticallyactuated bubbles in oil as microrobots [12]. Varel recently described a rotating table based on water droplets moving unidirectionally along a circular track micromachined onto a substrate subject to vertical oscillations [13]. A low-voltage microgripper utilizing a liquid droplet to pick up and release micro-objects is presented in [14]. We call microrobotics enabled by drops and bubble drop-botics or drobotics.

Soft robotics takes inspiration from animals in the way it attempts to develop robots with soft and flexible structures. This gives the robots additional degrees of freedom and eases gaits change. Robots can thus operate in unstructured environments and limited spaces [15]. Recently, Martinez combined this approach with preliminary works on compliant actuators based on flexible elastomers [16]. Flexible medical instruments are reviewed in [17].

The link between compliant and bubble-based actuators is their use of a confining membrane to store the actuation pressure-either polymeric, or directly provided by the liquid-gas interface). Bubble robots therefore represent a possible way toward miniaturization of compliant actuators.

After recalling the physics involved in supporting a table by surface tension forces (Sect. II), the present contribution introduces Drobot, a new platform design that allows for manipulation in a gaseous environment (Sect. III). In the new design the generation of droplets (i.e. liquid bridges) can be driven by a controller. Experimental results including kinematic (output/input ratio), static (compliance) and dynamic (response time and evaporation time) characterizations of the platform are then presented in Sect. IV, as well as preliminary, promising trials with an ionic liquid, whose negligible evaporation rate is suitable for vacuum or hot environments. Preliminary results are also shown towards a method to deduce the platform attitude from the measurement of droplets' electrical resistance. Conclusions are finally drawn towards the automatic control of the platform (Sect. V), which is however beyond the scope of this contribution.

\section{PhysicAl PRINCIPLES}

The developments hereby described are enabled by the scaling laws of surface tension effects [3]. Such effects scale linearly with the length scale as they depend on the surface 
tension parameter $(\gamma$, in $\mathrm{N} / \mathrm{m})$. Therefore, in the domain of microsystems-i.e. below the so-called capillary length (typically a few $\mathrm{mm}$ ) - they overcome gravity and inertia. Surface tension effects take place at liquid-gas interfaces, and they are twofold. First, the Laplace law relates the mean curvature $H$ and the surface tension of the interface to a pressure difference $\Delta p$ across it :

$$
\Delta p=2 H \gamma
$$

Second, tension forces act on the solids wet by a liquid-gas interface. Such forces are located on the triple contact line of the liquid on the solid. They are directed tangentially to the interface, always in the direction of the liquid.

In spite of their favorable scaling law, a known weakness of surface tension effects is the lack of repeatability induced by liquid evaporation. In this work, we show that ionic liquids can be used to cope with this issue (Sect. IV-E).

\section{DROBOT DESIGN}

\section{A. Overview}

Fig. 1 shows the CAD model of Drobot. The device is composed of a trefoil-shaped table (1) supported by three droplets (2). The droplets are generated by ejection from an underlying platform (3). The table can be translated vertically with respect to the platform and rotated about two axes parallel to the platform plane. Three degrees of freedom can thus be actuated, similarly to a three-leg parallel robot.

A droplet is formed by ejecting a certain volume of water from a tank (4). The tank is closed off by a flexible silicone membrane (5). A linear actuator adjoined to the membrane (6) deforms it so as to push liquid out of the tank up to the platform through the water circuit (7).

The droplets act as compliant actuators and lift the table thanks to surface tension effects (Sect. II). The resulting force applied on the table is due to the Laplace pressure difference $\Delta p$ (upwards, repulsive force for $H>0$, and conversely) and to the tension force along the triple line (downwards, attractive force). Thus, when the droplet is compressed, a repulsive force is exerted on the table. Conversely, when the droplet is stretched, an attractive force acts on it. This mechanical behavior is similar to that of a spring [6].

\section{B. Design and Manufacturing}

The design of Drobot was oriented towards ease of use and compactness. The platform which generates the droplets is composed of a water tank, a flexible silicone membrane, a set of electrodes and various supports.

The water tank features three channels manufactured using moulding techniques. The channels are shaped to avoid trapping bubbles inside the tank. Each channel is closed off by a flexible silicone membrane (Axson Essil 291, Young's modulus of $1.7 \mathrm{MPa}$, thickness of $700 \mu \mathrm{m}$ ). Sealing between channels and membrane needs to be liquidproof.

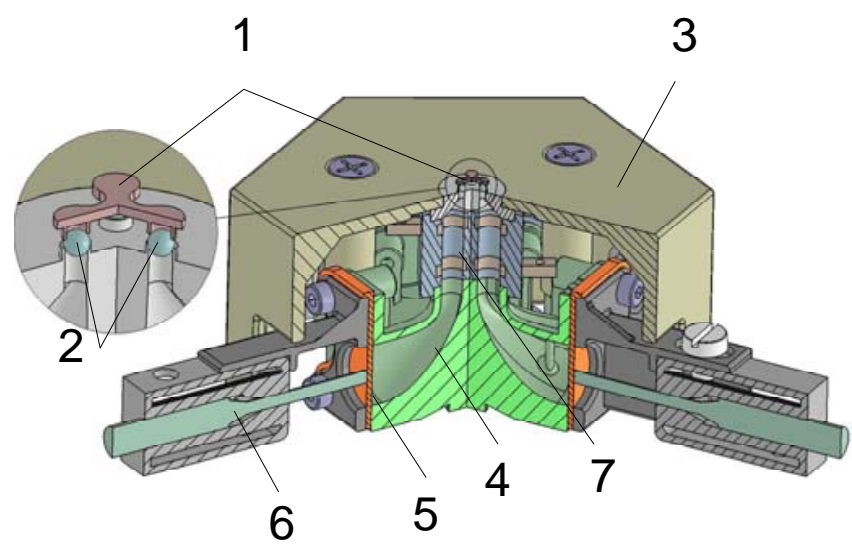

Fig. 1: CAD model of the Drobot platform (elements 1-7 are described in the text)

\section{Position Sensor}

A measurement method for tracking the position of the table has been developed by [18]. It relies on measuring the electrical resistance of each droplet to deduce the respective height. The present design includes a set of electrodes to perform this function. In the robot analogy, the electrodes constitute compact, integrated proprioceptive sensors that can measure the length of each robot leg.

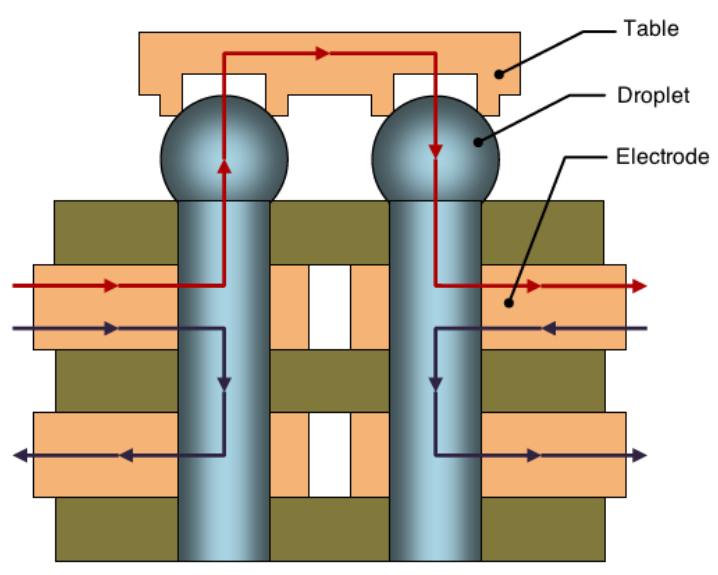

Fig. 2: Sketch of the electrical circuits embedded in Drobot for position measurement (not to scale). For each pair of channels (only one pair represented here), the bottom (blue) electrical circuit allows the liquid resistivity calibration, and the top (red) electrical circuit allows the determination of the droplets height from their electrical resistance.

\section{EXPERIMENTAL RESULTS}

Fig. 3 illustrates the experimental setup used for the characterizations of Drobot described in this section.

\section{A. Kinematics}

A first characterization of the platform related the input displacement $u$ imposed to the membrane by a single actuator to the output vertical displacement (quantifying tilt) of 


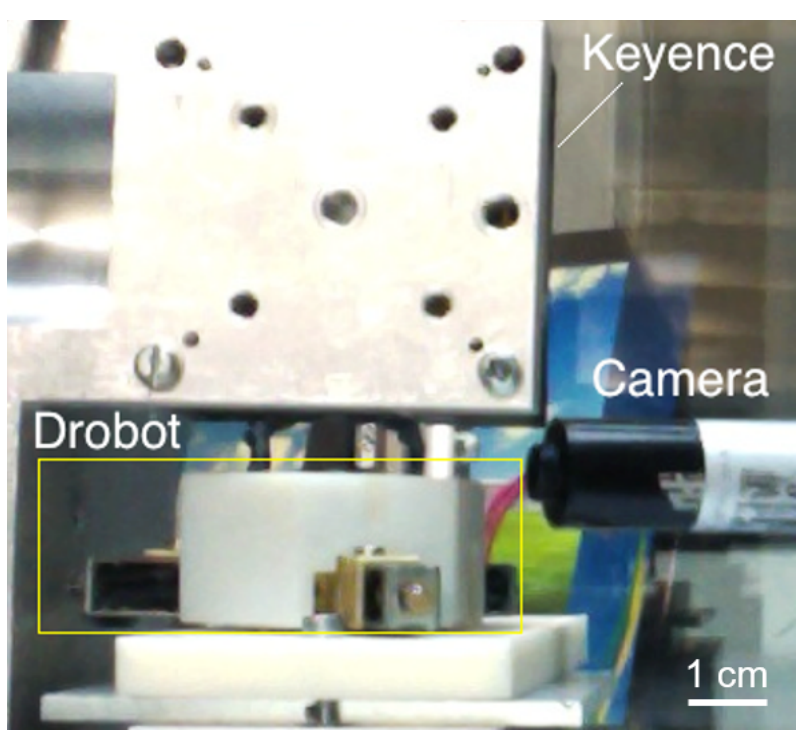

Fig. 3: Experimental setup : the Drobot platform, an LC-2440 Keyence laser atop to measure the position of the table, and a side camera to image drops and liquid menisci.

the table $z$. To determine the output/input ratio $\alpha=z / u$, an input signal was applied to one of the three actuators and the corresponding table position was recorded (Fig. 4a). As it can be seen, evaporation of the meniscus produced a slow decrease of the table position. Still, the table position amplitude was measured to be about 6 times the actuator step amplitude, corresponding to an amplification of the input position signal by a factor $\alpha=6$. As shown in Fig. 4b, this ratio is constant for liquid volumes ranging from 0.02 to $0.002 \mu \mathrm{L}$. The measurements were repeatable within an accuracy of $\pm 5 \mu \mathrm{m}$.

As a consequence, the platform is able to amplify the input position independently of the volume of liquid.

\section{B. Membrane Characterization}

As explained previously, one key advantage of the proposed platform is its compliance. The source of such compliance is twofold : partly coming from surface tension, and partly from the membrane stiffness. The complete compliance (or its inverse, the stiffness) must be known in order to chose suitable actuators.

The stiffness induced by the membrane can be obtained by relating the force increment $\mathrm{d} F$ developed by the actuator to deform the membrane by an extra displacement $\mathrm{d} u$ :

$$
\mathrm{d} F=k_{m} \mathrm{~d} u
$$

where $k_{m}$ is the membrane stiffness. This material response was experimentally measured through the principle depicted in Fig. 5. A cantilevered beam with known stiffness $k=$ $20 \mathrm{~N} / \mathrm{m}$ was pushed against the membrane with an actuator whose displacement $a$ was recorded. Simultaneously, the deflection $\delta$ of the cantilevered beam was measured, which provided the actual membrane displacement $u=a-\delta$. The applied force corresponding to $u$ is given by $k \delta$. In
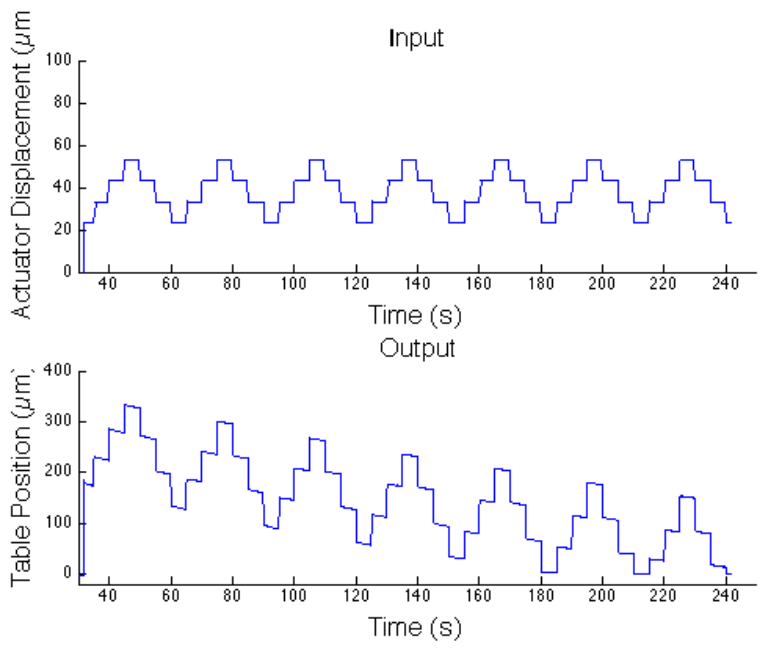

(a)

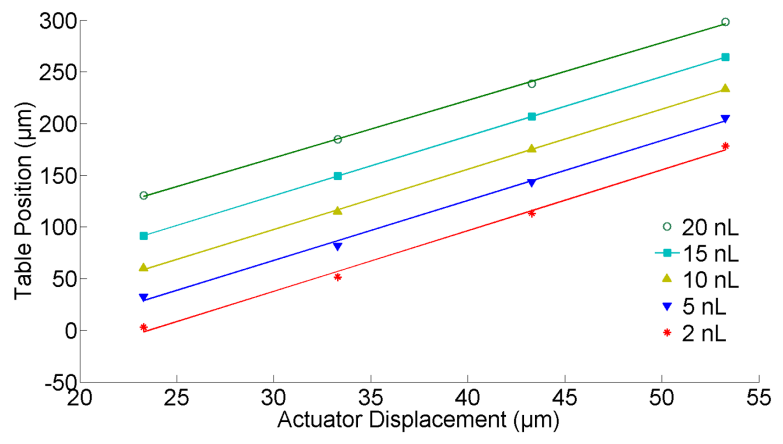

(b)

Fig. 4: Kinematic ratio between single actuator displacement (input) and vertical (z) table position (output). (a) Input and output signals, (b) the relationship has constant slope (about $6 \mu \mathrm{m} / \mu \mathrm{m}$ ) for liquid volumes ranging from 20 to $2 n \mathrm{~L}$.

case of linear response of the membrane, the slope of its characteristics would correspond to its stiffness $k_{\mathrm{m}}$.

The experimental results are shown in Fig. 6. For $a>$ $500 \mu \mathrm{m}$, the deflection $\delta$ becomes equal to a (Fig. 6a, red part), which means that the membrane displacement increment is equal to zero : $\Delta u=\Delta a-\Delta \delta=0$. We will limit our analysis to a single actuator displacement $a=420 \mu \mathrm{m}$ (solid triangle symbols). Experimentally, this corresponds to $u=200 \mu \mathrm{m}$, i.e. a droplet height increase $z=6 u=1.2 \mathrm{~mm}$, which is a conservative assumption. These results are used to compute the applied force $k \delta$ as a function of $u$ (Fig. 6b), leading to a linear membrane behaviour in our domain of interest (i.e. $u<200 \mu \mathrm{m}$ ), with a stiffness $k_{\mathrm{m}}=14 \mathrm{~N} / \mathrm{m}$.

However, we must also consider the stiffness $k_{c}$ induced by the meniscus. The capillary stiffness of a spherical cap can be roughly estimated as follows. Let us consider the droplet shown in Fig. 7. In the depicted situation with cap height $z$, the curvature radius $R$ is given by :

$$
R(z)=\frac{D^{2}+4 z^{2}}{8 z}
$$




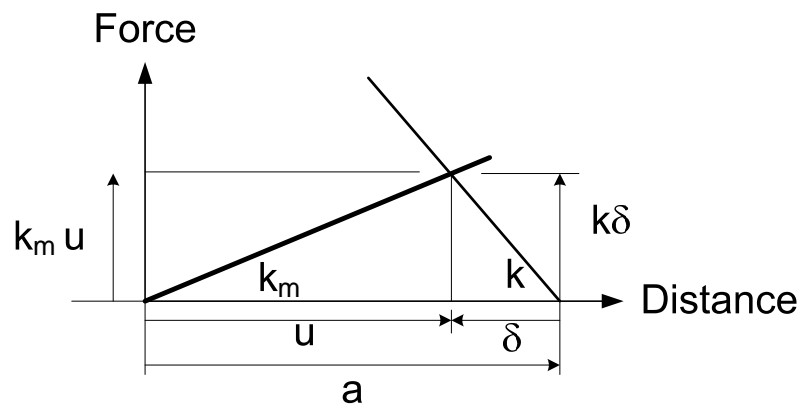

Fig. 5: Measurement principle of the membrane stiffness.

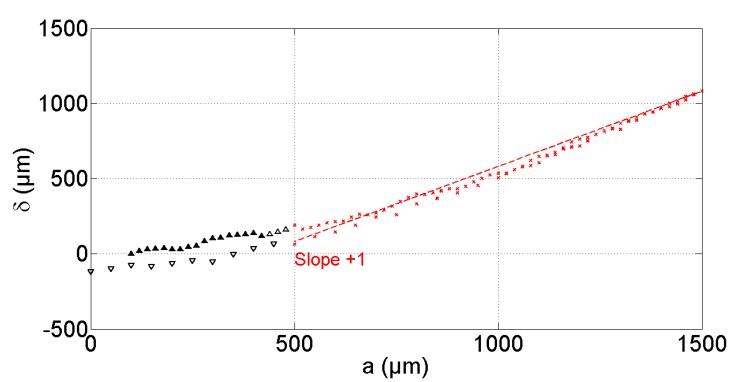

(a)

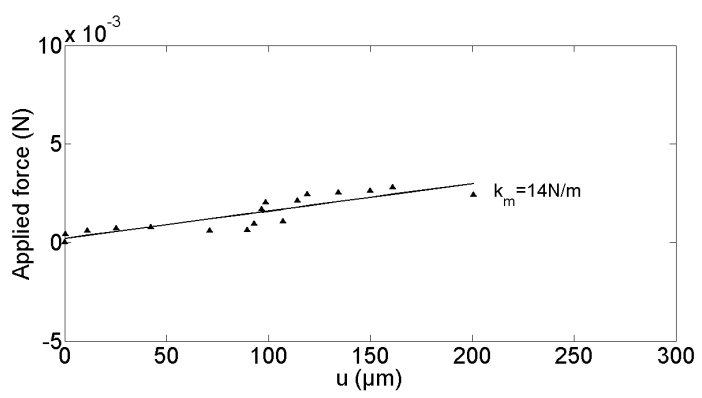

(b)

Fig. 6: (a) Deflection $\delta$ of our cantilever-based force sensor as a function of single actuator displacement $a$; (b) Forcedisplacement characteristics of the membrane, in the range of interest $u=0-200 \mu \mathrm{m}$

leading to a capillary pressure $p(z)$ in the drop (and the connected reservoir in the Drobot) given by :

$$
p(z)=\frac{2 \gamma}{R(z)}=\frac{16 \gamma z}{D^{2}+4 z^{2}}
$$

The pressure energy $E$ stored in the liquid is thus $E=p V$, where $V$ is the volume of liquid in the Drobot. When the input $u$ varies, so does $z$, which gives a force and a capillary stiffness defined as $F(u)=\mathrm{d} E / \mathrm{d} u$ and $k_{c}=\mathrm{d}^{2} E / \mathrm{d} u^{2}$, respectively :

$$
\begin{aligned}
F(u) & =\frac{16 \alpha \gamma V}{D^{2}} \frac{1-(2 \alpha u / D)^{2}}{\left(1+(2 \alpha u / D)^{2}\right)^{2}} \\
k_{c}(u) & =\frac{64 \alpha^{2} \gamma V}{D^{3}} \frac{(2 \alpha u / D)\left((2 \alpha u / D)^{2}-3\right)}{\left(1+(2 \alpha u / D)^{2}\right)^{3}}
\end{aligned}
$$

For small displacement $u$, the latter equation can be further simplified into $k_{c} \approx-\left(384 \alpha^{3} \gamma V / D^{4}\right) u$. Interestingly $k_{c}$ is

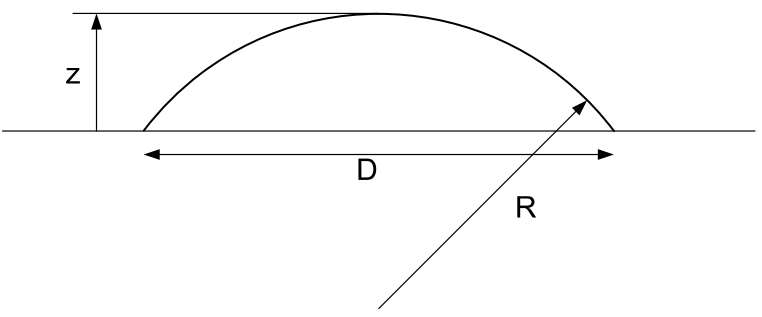

Fig. 7: Droplet shaped into a spherical cap of foot diameter $D$, height $z$ and curvature radius $R$. In Drobot, $D=1 \mathrm{~mm}$.

negative because $R$ decreases when $u$ (i.e. $z$ ) increases (see Fig. 7). This opens interesting perspectives from a control point of view. For Drobot, similar developments will need be led not for drops as shown in Fig. 7 but for menisci supporting the table.

\section{Dynamic Response}

In order to estimate the table dynamics, the system response to a step input displacement is shown in Fig. 8. Before the step, a slow evaporation-driven decrease is observed (slope of about $0.8 \mu \mathrm{m} / \mathrm{s}$ ). The step is triggered at time $t=0$, and a rising time $\tau=100 \mathrm{~ms}$ is measured. The response curve was fitted with the following equation :

$$
\text { Position }_{\text {Table }}=A\left(1-\mathrm{e}^{-\frac{t}{\tau}}\right)
$$

After the step, a linear decrease is observed again, with a decrease rate of about $0.8 \mu \mathrm{m} / \mathrm{s}$ (after $t=2 \mathrm{~s}$ ).

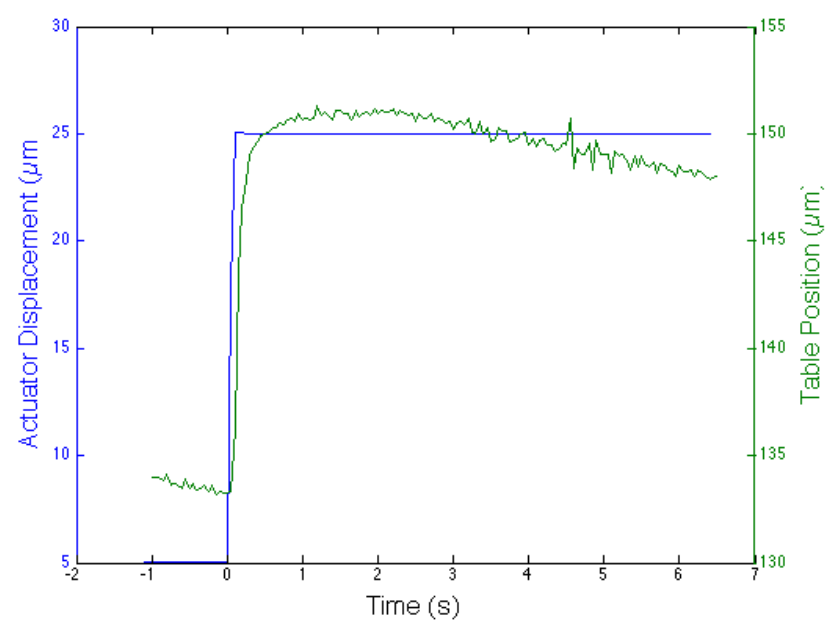

Fig. 8: Step response. Two different time scales are observed : background evaporation, at a rate of about $-0.8 \mu \mathrm{m} / \mathrm{s}$, and rising time of the input step of about $\tau=100 \mathrm{~ms}$.

The existence of two different time scales for evaporation and step response opens perspective towards a feedforward control technique to compensate droplet evaporation.

\section{Position Measurement}

Fig. 9 reproduces the proof-of-concept reported in our preliminary work [18]. A linear relationship between the 
droplet height and its electrical resistance for a given volume of liquid can be appreciated. Therefore, after volume calibration, the electrical resistance could be used to measure the height. This would provide a compact and real-time sensing method towards the control of the table position.

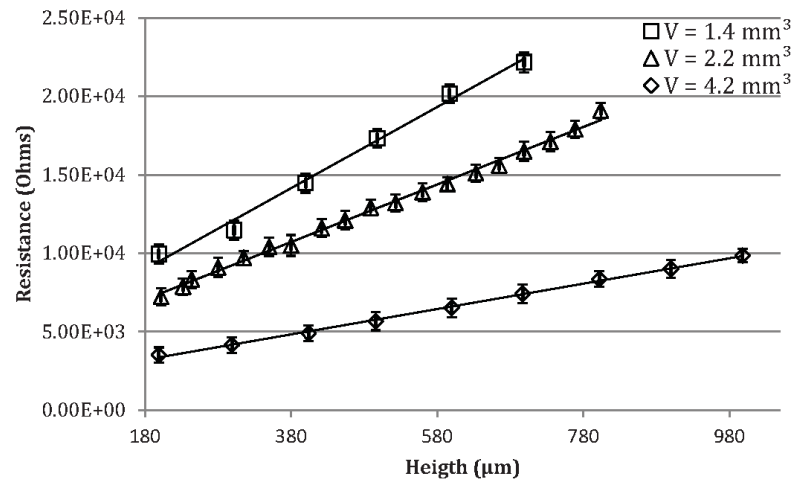

Fig. 9: Droplet resistance as a function of its height for increasing volumes of liquid. The linear relationship opens perspectives to measure the droplet height from its electrical resistance. (Reprinted from [18], in accordance with the MDPI Open Access Information and Policy)

\section{E. Use of Ionic Liquids}

Ionic liquids are molten salts at ambient temperature. Their properties are interesting for microsystems engineering. Due to very low vapor pressure, they exhibit almost no evaporation and are moreover suitable for vacuum environments or working temperatures larger than $100^{\circ} \mathrm{C}$. Their use in fluidic assembly was recently reported by $\mathrm{Al}$ Amin [19].

Ionic liquids are composed by a cation and an anion. We synthetized a cation very similar to C10MI (ID 239 according to Zhang's classification [20]), and the TFSI anion shown in Fig. 10 (ID 031). Density and dynamic

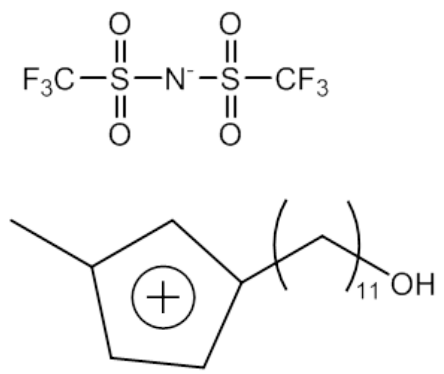

Fig. 10: Chemical structure of the synthesized TFSI anion.

viscosity of this liquid are tabulated by Zhang at about $\rho=1.27 \mathrm{~g} / \mathrm{cm}^{3}$ and $\eta=153 \mathrm{mPas}$. We could therefore measure its surface tension with the pendant drop method at about $\gamma=37.7 \mathrm{mNm}^{-1}$. This value is corroborated at about $\gamma=39.7 \mathrm{mNm}^{-1}$ by a capillary force measurement performed with a dedicated setup, whereby the capillary force between a flat plane and a sphere is measured as well as the respective contact angles, and the surface tension is deduced using the Israelachvili equation [21].

Since the surface tension of our ionic liquid is smaller than that of water, we investigated whether the drops are strong enough to support the table, and whether the platform can withstand eventual ionic liquid spreading.

The answer to the first question is positive, as shown in Fig. 11a. Our setup was however at its limits of droplet confinement [22] (Fig. 11b). A new design should include sharper edges or more hydrophobic materials to better confine the spreading, low-surface-tension ionic liquid.
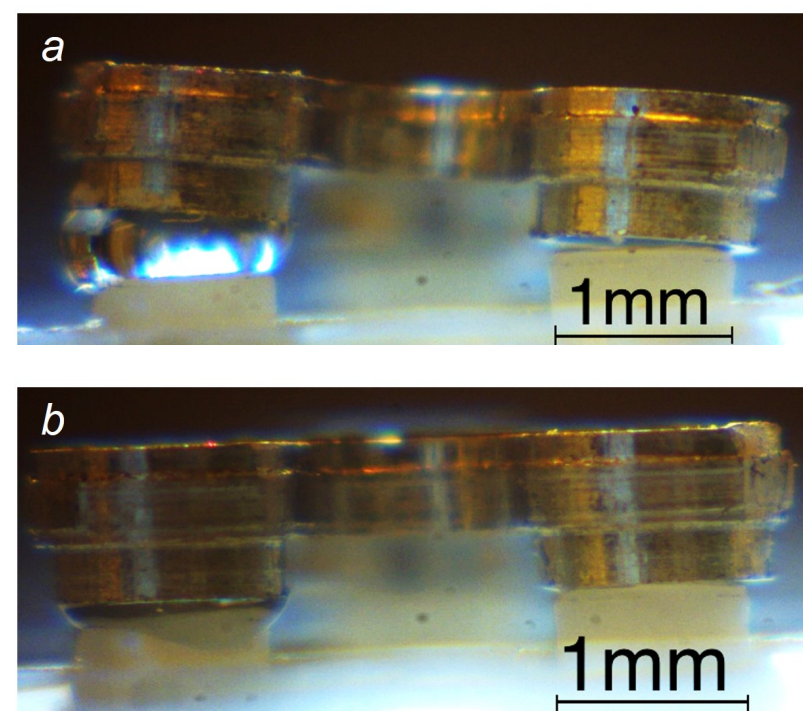

Fig. 11: Use of ionic liquid. (a) Table supported by a single drop. (b) The liquid bridge is not sufficiently constrained by the geometry of the substrate [22], and the ionic liquid overflows and spreads onto the platform.

The interesting property of such liquids is however their negligible evaporation rate, as qualitatively shown in Fig. 12.
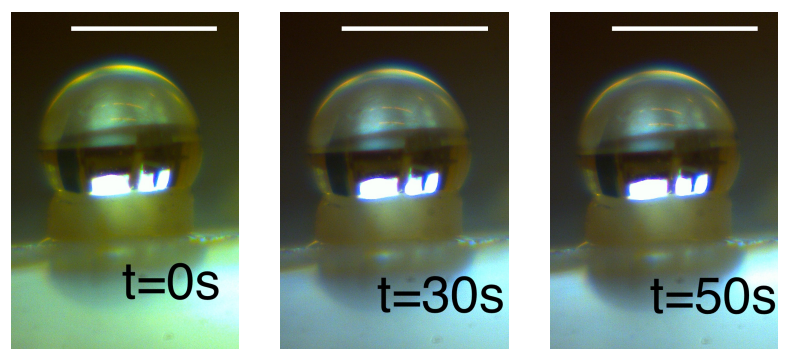

Fig. 12: Sequential snapshots of a $0.76 \mu$ lionic liquid droplet at $t=0,30$ and $50 \mathrm{~s}$, evidencing the absence of significant evaporation. Scale bars are $1 \mathrm{~mm}$.

The difference in evaporation rate between the ionic liquid and water is quantified in Fig. 13. The height of the ionic liquid droplet did not vary after $300 \mathrm{~s}$, while that of the water droplet decreased linearly with a rate of about $-0.97 \mu \mathrm{m} / \mathrm{s}$. 


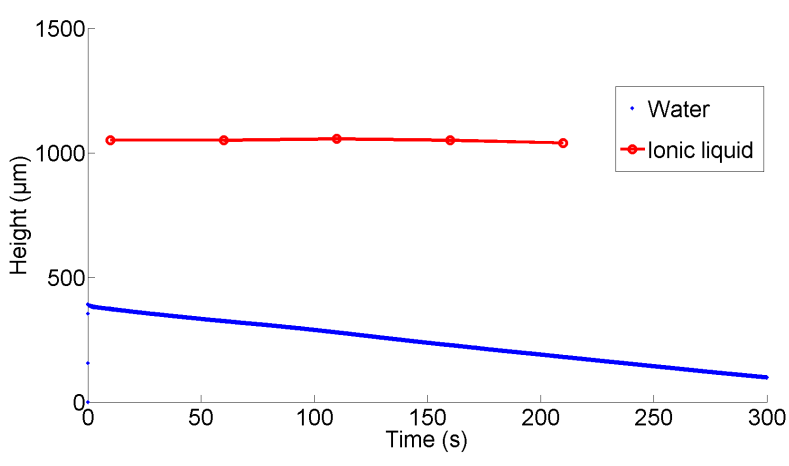

Fig. 13: Time variation of liquid droplet height in air.

\section{CONCLUSiOnS}

This paper introduced Drobot, a droplet-actuated platform for compliant micromanipulation. Physical principles, design and manufacturing of the prototype were presented. Experimental characterization of kinematics, compliance-arising from both flexible silicone membrane and liquid meniscusand dynamics along a single degree of freedom of the platform were reported. Among other results, an output/input displacement ratio of $6 \mu \mathrm{m} / \mu \mathrm{m}$, a response time of $\tau=$ $100 \mathrm{~ms}$ and water droplet evaporation rate of $-0.8 \mu \mathrm{m} / \mathrm{s}$ were established. To cope with output displacement drifts caused by liquid evaporation, an ionic liquid was tested obtaining encouraging results. The liquid has negligible volatility and is thus particularly suitable for vacuum or hot environments. Yet effective confinement over the platform of the highly wetting ionic liquid needs to be enforced for stable support of the table. Moreover, a technique to estimate the height of liquid droplets through the measurement of their electrical resistivity was proposed. Such electrical proprioceptive sensing is compatible with and expected to enable an integrated closed-loop control of the platform attitude.

This work contributes toward the automatic control of the platform and its effective use in microassembly. Future works on Drobot will focus on reduction of droplet evaporation using ionic liquid, characterization of all degrees of freedom and implementation of proprioceptive position sensors through droplet resistance measurement.

\section{ACKNOWLEDGMENT}

This work originates in Dr. Cyrille Lenders's $\mathrm{PhD}$ in collaboration with Dr. Michael Gauthier. Many colleagues contributed to the development of the concept : beside his co-authors cited in this work, we recall the appreciated help of P. Rougeot, D. Guibert, and B. Tartini. The project has been supported over its development by Wallonie Bruxelles International (WBI, Tournesol grant, MOMIE projet), CNRS, Lycée Technique Edgar Faure (Morteau, France), and recently, by the Labex ACTION project (contract "ANR-11LABX-0001-01") and the IAP 7/38 MicroMAST funded by BELSPO (Belgian Science Office Policy).

\section{REFERENCES}

[1] P. L. C. Lenders, M. Gauthier, "Microbubble generation using a syringe pump," in IEEE Int. Conf. Intell. Robots Syst., St. Louis (USA), 2009, pp. 1395-1400.

[2] M. Gauthier and S. Régnier, Eds., Robotic Micro-Assembly. WileyIEEE press., 2010.

[3] P. Lambert, Ed., Surface Tension Effects in Microsystems : Engineering Below the Capillary Length. Springer, 2013.

[4] C. Clévy, A. Hubert, and N. Chaillet, "Flexible micro-assembly system equiped with an automated tool changer," J. Micro-Nano Mech., vol. 4, pp. 59-72, 2008.

[5] P. M. Moore, M. Rakotondrabe, C. Clévy, and G. J. Wiens, "Development of a modular and compliant microassembly platform with integrated force measurement capabilities," in 7th International Conference on MicroManufacturing, INCOMM'12., Chicago (USA), 2012.

[6] C. Lenders, M. Gauthier, R. Cojan, and P. Lambert, "Three-dof microrobotic platform based on capillary actuation," IEEE Trans. Robot, vol. 28, pp. 1157-1161, 2012.

[7] W. Dong, M. Gauthier, C. Lenders, and P. Lambert, "A Gas Bubblebased Parallel Micro Manipulator : Conceptual Design and Kinematics Model," J.Micromech. Microeng., vol. 22, pp. 1-11, 2012.

[8] N. Crane, O. Onen, J. Carballo, Q. Ni, and R. Guldiken, "Fluidic assembly at the microscale : progress and prospects," Microfluid. Nanofluid, vol. 14, pp. 383-419, 2013.

[9] K. Ryu, J. Zueger, S. K. Chung, and S. K. Cho, "Underwater propulsion using ac-electrowetting-actuated oscillating bubbles for swimming robots," in 23rd IEEE Int. Conf. on Micro Electro Mechanical Systems (MEMS 2010), Hong-Kong (China), January 2012, pp. 160163.

[10] J. Feng and S. Cho, "Micro propulsion in liquid by oscillating bubbles," in 26th IEEE Int. Conf. on Micro Electro Mechanical Systems (MEMS 2013), Taipei (TW), January 2013, pp. 63-66.

[11] W. Hu, Q. Fan, and A. Ohta, "An opto-thermocapillary cell micromanipulator," Lab on a Chip, vol. 13, pp. 2285-2291, 2013.

[12] K. Ishii, W. Hu, and A. Ohta, "Cooperative micromanipulation using optically controlled bubble microrobots," in IEEE Int. Conf. on Robotics and Automation (ICRA), Saint-Paul Minnesota (USA), May 14-18 2012.

[13] C. Varel and K. F. Böhringer, "Liquid droplet micro-bearings on directional circular surface ratchets," in 27th IEEE Int. Conf. on Micro Electro Mechanical Systems (MEMS), San Francisco (CA), January 26-30 2014, pp. 983-986.

[14] A. Vasudev, A. Jagtiani, L. Du, and J. Zhe, "A low-voltage droplet microgripper for micro-object manipulation," Journal of Micromechanics and Microengineering, vol. 19, no. 7, p. 075005, 2009.

[15] D. Trivedi, C. D. Rahn, W. M. Kier, and I. D. Walker, "Soft robotics Biological inspiration, state of the art, and future research," Appl. Bion. Biomech., vol. 5, pp. 99-117, 2008.

[16] R. Martinez, J. Branch, C. Fish, L. Jin, R. Shepherd, R. Nunes, Z. Suo, and G. Whitesides, "Robotic tentacles with three-dimensional mobility based on flexible elastomers," Adv. Mat., vol. 25, pp. 205-212, 2013.

[17] A. D. Greef, P. Lambert, and A. Delchambre, "Towards flexible medical instruments : Review of flexible fluidic actuators," Precision Engineering, vol. 33, pp. 311-321, 2009.

[18] R. Casier, C. Lenders, M. Sausse, M. Gauthier, and P. Lambert, "Position measurement/tracking comparison of the instrumentation in a droplet-actuated-robotic platform," Sensors, vol. 13, pp. 5857-5869, 2013.

[19] A. A. Amin, A. Jagtiani, A. Vasudev, J. Hu, and J. Zhe, "Soft microgripping using ionic liquids for high temperature and vacuum applications," J. Micromech. Microeng., vol. 21, p. 125025, 2011.

[20] S. Zhang, N. Sun, X. He, X. Lu, and X. Zhang, "Physical properties of ionic liquids : Database and evaluation," J. Phys. Chem. Ref. Data, vol. 35, no. 4, pp. 1475-1517, 2006.

[21] P. Lambert, Ed., Capillary forces in microassembly. Springer, 2007.

[22] M. Mastrangeli, J.-B. Valsamis, C. van Hoof, J.-P. Celis, and P. Lambert, "Lateral capillary forces of cylindrical fluid menisci : a comprehensive quasi-static study," J. Micromech. Microeng., vol. 20, p. 075041, 2010. 